\title{
Role of a paracrine action of mesenchymal stem cells in the improvement of left ventricular function after coronary artery occlusion in rats
}

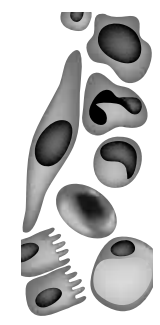

\author{
WengdeDai', \\ Sharon L Hale' \& \\ Robert A Kloner't \\ ${ }^{\dagger}$ Author for correspondence \\ ${ }^{1} \mathrm{~T}$ he H eart Institute, Good \\ Samaritan H ospital, Division \\ of Cardiovascular M edicine of \\ the Keck School of M edicine \\ at U niversity of Southern \\ California, 1225 Wilshire \\ Boulevard, L os Angeles, \\ CA 90017-2395, USA \\ Tel.: +1 2139774050 ; \\ Fax: +1 2139774107 \\ E-mail: Rkloner@ \\ goodsam.org
}

\begin{abstract}
Purpose: We aimed to determine whether soluble factors released by cultured mesenchymal stem cells (MSCs) improved cardiac function in an experimental model of myocardial infarction. Methods: MSCs were cultured in fresh medium. The conditioned medium, which contained factors secreted by MSCS, was collected after 4 days of culture. Fischer rats with 1-week-old myocardial infarction were divided into four groups that received: saline $(n=12)$; fresh medium $(n=10)$; conditioned medium $(n=8)$; or 2 million MSCs in fresh medium $(n=10)$ by direct intramyocardial injection. A total of 4 weeks later, left ventricular (LV) function was assessed by LV angiogram and by LV catheterization. Hearts were processed for histology. Results: Before treatment, LV angiogram assessment demonstrated that the baseline LV function was comparable among the four groups. At 4 weeks after treatment, LV angiogram and LV catheterization showed that LV ejection fraction was better in the fresh medium $(49.5 \pm 1.0 \%)$, conditioned medium $(48.5 \pm 2.1 \%)$ and MSCs groups $(49.9 \pm 4.2 \%)$ than in the saline group $(43.7 \pm 1.2 \% ; p<0.05)$. There were no significant differences in heart rate, blood pressure, postmortem LV volume, infarct size or septum thickness among the groups. The scar thickness was similar in the saline $(395 \pm 31 \mu \mathrm{m})$, fresh medium $(404 \pm 30 \mu \mathrm{m})$ and conditioned medium ( $397 \pm 34 \mu \mathrm{m})$ groups, but significantly thicker in the MSCs group $(560 \pm 51 \mu \mathrm{m} ; \mathrm{p}<0.05)$. Conclusion: Fresh medium, conditioned medium and MSC injection all improved LV function at 4 weeks after treatment compared with saline treatment in a rat myocardial infarct model; only MSCs increased wall thickness. Since the culture medium contains nutrients and bovine serum, the roles of the soluble factors released by MSCs might be masked. The effect of these nutrients needs further investigation.
\end{abstract}

A paracrine effect of transplanted mesenchymal stem cells (M SCs) has been suggested to play a crucial role in the improvement of cardiac function in an experimental myocardial infarction model. We assessed the effects of saline, fresh culture medium, MSC-derived conditioned medium and M SC s on the left ventricular (LV) function in a rat myocardial infarction model. The results showed that fresh medium, conditioned medium and MSCs all improved LV function at 4 weeks after treatment compared with saline treatment. The mechanisms of beneficial effects of culture medium remain unclear and need further investigation.

Bone marrow-derived M SC s are multipotenregeneration, celltransplantation therapy, mesenchymal stem cell, myocardial infarction, paracrine action

future ${ }_{\text {medicine }}^{\text {pasg }}$ fs tial cells, which have high self-renewal capability and are able to differentiate into a wide variety of lineages, including endothelial and myogenic cells [1]. Thus, M SC s are considered to be one of the potential cell sources for cell transplantation therapies of cardiac regeneration
[2]. Although animal experiments and clinical trials have demonstrated that M SC transplantation therapies improve cardiac function after myocardial infarction, the underlying mechanisms of these therapies remain unclear. G rafted M SC s have been reported to form clusters of cell grafts and express cardiac muscle proteins on the border of the infarct in a rat myocardial infarction model [3] and induce neovascularization [4]. O ur recently published data showed that M SC transplantation transiently improved LV function at 4 weeks after transplantation in a rat myocardial infarction model, when the grafted M SC s did not show complete myogenic differentiation and did not result in visible replacement of scar with sheets of muscle cells. The beneficial effect on LV function was lost at 6 months, although the grafted M SC s survived and expressed muscle markers and incorporated into blood vessels [5]. These results suggested that the improvement of cardiac function might 
be due to a paracrine effect of the transplanted M SC s. To address our hypothesis, in this study, we assessed the effects of conditioned medium from cultured M SC s on the LV function in a rat myocardial infarction model.

\section{Materials \& methods}

All animal studies were approved by the Institutional Animal $\mathrm{C}$ are and $\mathrm{U}$ se $\mathrm{C}$ ommittee of $\mathrm{G}$ ood Samaritan Hospital and comply with the Guide for the Care and Use of Laboratory Animals [6]. The Association for Assessment and Accreditation of Laboratory Animal Care International accredits G ood Samaritan H ospital.

\section{MSC isolation \& expansion}

Allogeneic MSCs were isolated, characterized and cultured according to the previous established methods [7,8]. Briefly, the M SC s were isolated from the femoral and tibial bones of donor $\mathrm{ACl}$ rats (a cross between the August and Copenhagen-Irish strains), as described previously [7,8]. MSCS were expanded in fresh medium composed of $44.5 \% \mathrm{H}$ am's $\mathrm{F} 12$ medium (F12), 44.5\% minimum essential medium (MEM), 10\% fetal bovine serum and $0.1 \%$ antibiotic/antimycotic. The conditioned medium was collected at 4 days after M SC s culture. All cells and medium were kept frozen until use. M SC viability ranged from 90 to $95 \%$, as assessed by trypan blue.

\section{Surgical preparation}

Female Fischer $C D F$ rats were anesthetized with intraperitoneal ketamine $(75 \mathrm{mg} / \mathrm{kg}$ ) and xylazine $(5 \mathrm{mg} / \mathrm{kg})$, intubated and mechanically ventilated with room air (rate 60 cycles/min, tidal volume $1 \mathrm{ml}$ per $100 \mathrm{~g}$ body weight, $\mathrm{H}$ arvard Apparatus Rodent Ventilator, M odel 683, M A, USA). The heart was exposed by a left thoracotomy through the fourth intercostal space. The free wall of the left ventricle was exposed after the pericardium was excised. The left anterior descending coronary artery was encircled with a silk suture and ligated. The rats recovered under care, and buprenex $(0.001 \mathrm{mg} / 100 \mathrm{~g}$ body weight, daily) was administered for 2 days as analgesic.

The rats with 1-week-old myocardial infarction were re-anesthetized and hearts re-exposed (as described above). After locating the infarcted area, MSC s $\left(2 \times 10^{6}\right.$ cells in $\sim 70 \mu \mathrm{l}$ fresh medium; $n=10)$, conditioned medium $(\sim 70 \mu$ l; $n=8)$, fresh medium alone $(\sim 70 \mu \mathrm{l} ; \mathrm{n}=10)$ or saline $(\sim 70 \mu \mathrm{l} ; n=12)$ were injected directly into the scar area with a 28-gauge needle attached to an insulin syringe. After successful injection, typified by the formation of a bleb covering the infarct zone, the chest was closed and animals recovered under care.

Assessment of LV function by angiography Left ventricular contrast angiography was performed with a XiScan $1000 \mathrm{C}$-arm X-ray system (XiTec, Inc; 3-inch field of view) before treatment at 1 week after induction of myocardial infarction at baseline and at 4 weeks after treatment. After rats were anesthetized, a catheter was inserted into the left jugular vein, followed by the injection of $1 \mathrm{ml}$ nonionic contrast. Under view of anterior-posterior and lateral projections, video images were acquired on half-inch super-VHS videotape at 30 frames/second under constant fluoroscopy. LV volumes in systole and diastole were calculated from the video images in a blinded manner. All parameters were averaged over three consecutive cycles in both projections. Ejection fraction (\%) was calculated as:

$$
100 \times \frac{\text { vol. in diastole }- \text { vol. in systole }}{\text { vol. in diastole }}
$$

and averaged over both projections.

Hemodynamic measurements

After angiography, a $2 \mathrm{~F}$ high-fidelity, cathetertipped micromanometer (model SPR-869, M illar, Inc) was inserted into the right carotid artery to record arterial blood pressure and heart rate, then advanced into the ascending aorta and into the LV to record peak maximum and minimum LV pressure change over time (dP/dt).

\section{Postmortem LV volume \& histological measurements}

After the completion of the hemodynamic measurement, hearts were arrested in diastole by an intravenous injection of $2 \mathrm{mEq} \mathrm{KCl}$ administered under deep anesthesia. The excised hearts were pressure-fixed with formalin (pressure equal to $13 \mathrm{~cm}$ water column). After fixation, postmortem LV volumes were measured by filling the cavity with water and weighing, repeated three times.

Following LV volume measurement, the hearts were cut into three transverse slices and embedded in paraffin and processed for histology. $\mathrm{H}$ ematoxylin and eosin, picrosirius red staining and immunohistochemical staining with primary antibody against sarcomeric actin (Dako M 0874, 1:75) were performed on sections ( $5 \mu \mathrm{m}$ thickness) of 
the paraffin-embedded tissue. Computerized planimetry of the histological images of the stained sections was used to measure and calculate:

- Scar thickness (average of five equidistant measurements) and septum thickness (average of three equidistant measurements);

- Epicardial circumference and endocardial circumference, and circumference occupied by the infarcted wall. The infarct size was expressed as percentage of total LV circumference;

- Expansion index was calculated as defined by $\mathrm{H}$ ochman and $\mathrm{C}$ hoo [9], which is expressed as: $\frac{\text { LV cavity area }}{\text { total LV area }} \times \frac{\text { Septum thickness }}{\text { Scar thickness }}$

\section{Statistics}

All data are presented as mean \pm standard error of the mean (SEM ). Comparisons of results among groups were made by one way AN OVA. If an $\mathrm{f}$ value of less than 0.05 was obtained, differences among the means were determined by Tukey's Studentized Range test.

Results

Two rats in the saline group and one in the fresh medium group died after anesthesia administration at 4 weeks after treatment. 0 ne in the saline group, two in the fresh medium group and one in the M SC group died after angiography. 0 ne in the saline group and one in the conditioned medium group were excluded for histology because of sample damage during processing.

\section{LV stroke volume \& ejection fraction by angiography}

The baseline LV stroke volumes and ejection fractions were comparable among the four groups before treatment at 1 week after myocardial infarction (Table1). At 4 weeks after treatment, the LV ejection fraction was similar in groups receiving fresh medium $(49.5 \pm 1.0 \%)$, conditioned medium $(48.5 \pm 2.1 \%)$ and M SCS $(49.9 \pm 4.2 \%)$, and was significantly greater than the group receiving saline (43.7 $\pm 1.2 \%$; $p<0.05)$ (Table1).

\section{Hemodynamics}

At 4 weeks after treatment, heart rate, blood pressure and -dP/dt (LV peak negative change in pressure over time) did not significantly differ between the four groups (Table1). +dP/dt (LV peak positive change in pressure over time) was similar in groups receiving conditioned medium and M SC s, but it was significantly greater in the fresh medium group compared with the group receiving saline $(p<0.05)$ (Table1).

Table 1. Parameters of left ventricular function and postmortem morphometery.

\begin{tabular}{lllll} 
& Saline group & Fresh medium & Conditioned medium & MSCs \\
Angiographic analysis $(\mathbf{n})$ & $\mathbf{1 0}$ & $\mathbf{9}$ & $\mathbf{8}$ & $\mathbf{1 0}$ \\
Stroke volume $(\mu \mathrm{l})$ at baseline & $120 \pm 4$ & $128 \pm 4$ & $132 \pm 3$ & $143 \pm 7$ \\
Stroke volume $(\mu \mathrm{ll})$ after treatment† & $129 \pm 6$ & $163 \pm 6$ & $170 \pm 6$ & $164 \pm 8$ \\
LVEF $(\%)$ at baseline & $54.7 \pm 1.2$ & $54.4 \pm 0.5$ & $53.4 \pm 1.6$ & $53.7 \pm 1.5$ \\
LVEF $(\%)$ after treatment & $43.7 \pm 1.2$ & $49.5 \pm 1.0^{*}$ & $48.5 \pm 2.1^{*}$ & $49.9 \pm 4.2^{*}$ \\
Hemodynamics $(\mathbf{n})$ & $\mathbf{9}$ & $\mathbf{7}$ & $\mathbf{8}$ & $\mathbf{9}$ \\
Heart rate (beats/minute) & $231 \pm 10$ & $227 \pm 10$ & $245 \pm 8$ & $219 \pm 7$ \\
Systolic blood pressure $(\mathrm{mmHg})$ & $145 \pm 7$ & $161 \pm 9$ & $158 \pm 6$ & $135 \pm 7$ \\
Diastolic blood pressure $(\mathrm{mmHg})$ & $109 \pm 3$ & $113 \pm 4$ & $117 \pm 2$ & $104 \pm 3$ \\
+dP/dt (mmHg/second) & $2746 \pm 700$ & $5350 \pm 627^{*}$ & $4895 \pm 296$ & $4769 \pm 625$ \\
-dP/dt (mmHg/second) & $1970 \pm 601$ & $3450 \pm 328$ & $2635 \pm 274$ & $3105 \pm 320$ \\
Postmortem analysis $(\mathbf{n})$ & $\mathbf{1 2}$ & $\mathbf{1 0}$ & $\mathbf{8}$ & $\mathbf{1 0}$ \\
Postmortem LV volume $(\mu \mathrm{ll})$ & $351 \pm 15$ & $379 \pm 14$ & $374 \pm 12$ & $367 \pm 22$ \\
Histology (n) & $\mathbf{1 1}$ & $\mathbf{1 0}$ & $\mathbf{7}$ & $\mathbf{1 0}$ \\
Infarct size, \% of LV circumference & $37.8 \pm 3.8$ & $39.7 \pm 3.1$ & $36.9 \pm 4.8$ & $33.6 \pm 4.3$ \\
Scar thickness $(\mu \mathrm{m})$ & $395 \pm 31$ & $404 \pm 30$ & $397 \pm 34$ & $560 \pm 51^{\ddagger}$ \\
Septum thickness $(\mu \mathrm{m})$ & $993 \pm 43$ & $1047 \pm 55$ & $1086 \pm 66$ & $1053 \pm 27$ \\
\hline
\end{tabular}

*Statistically significant vs saline, $\mathrm{p}<0.05$.

‡Statistically significant vs saline, fresh medium and conditioned medium, $\mathrm{p}<0.05$.

${ }^{\dagger}$ After treatment is 4 weeks after treatment.

$\mathrm{dP} / \mathrm{dt}$ : LV peak change in pressure over time; LV: Left ventricular; LVEF: Left ventricular ejection fraction; MSC: Mesenchymal stem cell. 


\section{Figure 1. Representative picrosirius red-stained sections of hearts show that cardiac muscle stains yellow, and collagen stains red.}
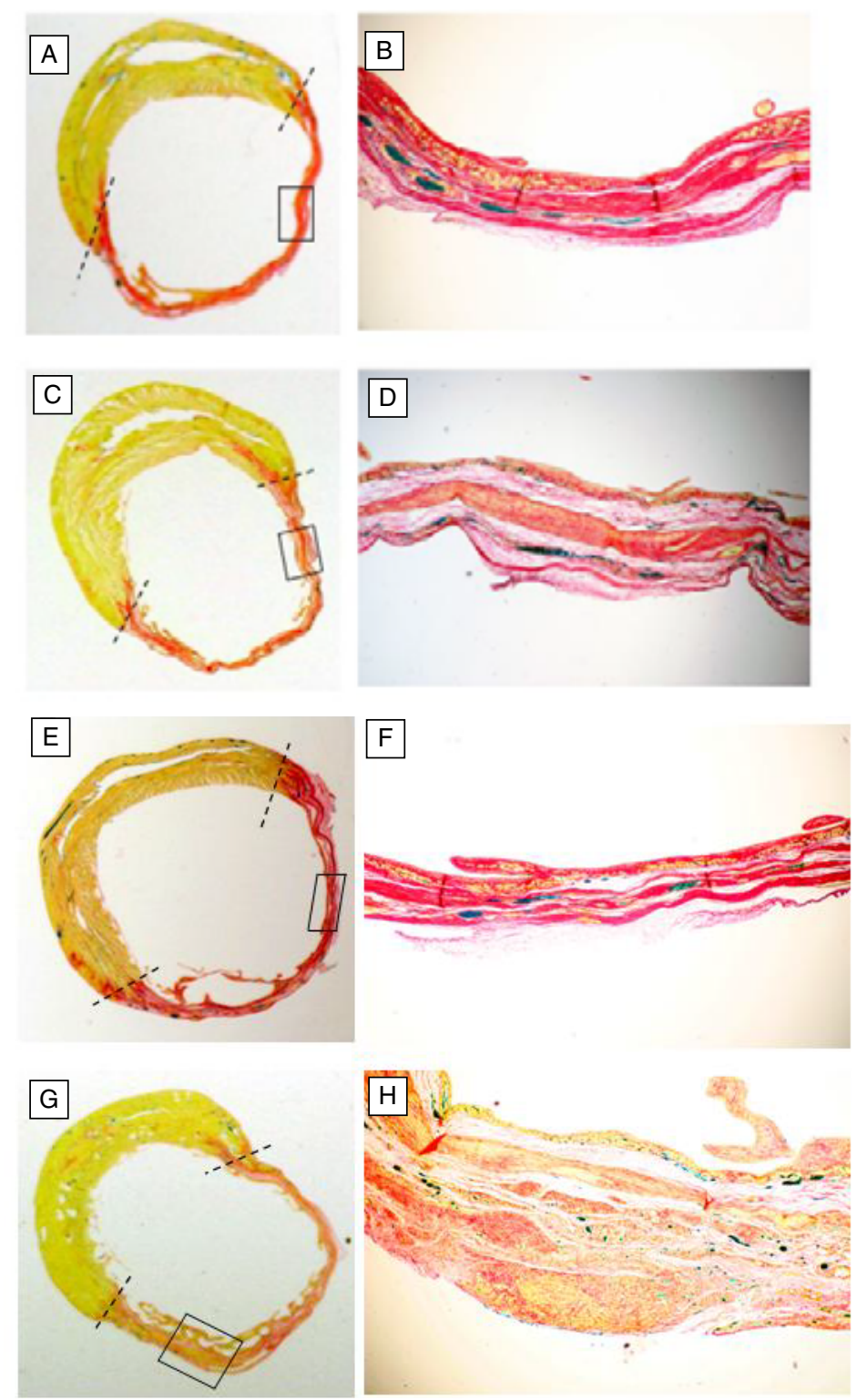

The blue staining shows the blood vessels. (B, D, F $\boldsymbol{\&} \mathbf{H}$ ) These are the higher magnification ( $\times 40$ ) of the boxed area in $\mathbf{A}$ (saline group), $\mathbf{C}$ (fresh medium group), $\mathbf{E}$ (conditioned medium group) and $\mathbf{G}$ (MSC group), respectively. The dotted lines show the border of myocardial infarction with thin-walled, transmural collagenous scars. Note that some areas of the MSC-treated scar area are thicker than in the other groups and have a higher cell density.

\section{Postmortem LV volumes \&} histological measurements At 4 weeks after treatment, there were no significant differences in the postmortem LV volume, infarct size as a percentage of the LV circumference or septum thickness among the 4 groups (Table1). The scar wall thickness was comparable in the groups receiving saline $(395 \pm 31 \mu \mathrm{m})$, fresh medium $(404 \pm 30 \mu \mathrm{m})$ and conditioned medium ( $397 \pm 34 \mu \mathrm{m}$ ), but was significantly thicker in the group receiving $\mathrm{MSC} S$ (560 $\pm 51 \mu \mathrm{m} ; \mathrm{p}<0.05)$ (Table1).

$\mathrm{H}$ ematoxylin and eosin, and picrosirius red staining showed that the scars were transmural and thin and composed of collagenous tissues with a thin discontinuous layer of subendocardial cardiac myocytes in all of the four groups (Figure1). M SC treatment increased scar thickness due to an increase in cell density, including cells that stained positive for $\alpha$-sacromeric actin besides an increase of collagen deposition (Figre2). The cell density in the infarct area was $4280 \pm 291$ cells $/ \mathrm{mm}^{2}$ in the M SC group. It was significantly higher than the cell density in the other three groups $\left(2124 \pm 116 \mathrm{cells} / \mathrm{mm}^{2}\right.$ in the saline group, $2112 \pm 101$ cells $/ \mathrm{mm}^{2}$ in the fresh medium group and $2267 \pm 119 \mathrm{cell} / \mathrm{s} / \mathrm{mm}^{2}$ in the conditioned medium group; $p<0.05$ ).

\section{Discussion}

This study demonstrates that allogeneic M SCS, fresh medium and conditioned medium all improve global LV function at 4 weeks after treatment. Combined transplantation with M SC s and fresh medium significantly increased scar thickness. These results suggest that there are some substances in the fresh medium that may benefit the grafted M SC s or the heart, and improve the LV function.

Cellular cardiomyoplasty aims to regenerate damaged myocardium and induce revascularization of the injured region through cell transplantation. Bone marrow contains a useful cell source for cell transplantation therapies $[10,11]$. Bone marrow-derived MSCs are multipotent cells and represent less than $0.01 \%$ of all nucleated bone marrow cells. Although they can be readily expanded in vitro, there is little evidence of in vivo proliferation due to contact inhibition [1]. Thus, M SCS need to be expanded ex vivo for cell therapy. The isolation and ex vivo expansion of M SC s are time consuming, making using of autologous cells difficult. The type of M SC that we used was allogenic and has been suggested to be immune-privileged [1]. Thus, an 'off-the-shelf' approach of allogeneic M SC s has the potential for clinical application.

MSC S are able to differentiate into cardiomyocytes in defined culture media [12]. Toma and colleagues transplanted human M SC s into normal left ventricle of CB17 SCID/beige adult mice and observed that the engrafted human 
Figure 2. Representative pictures to show the high density of cells in the scar area in the MSC-treated myocardial infarction.

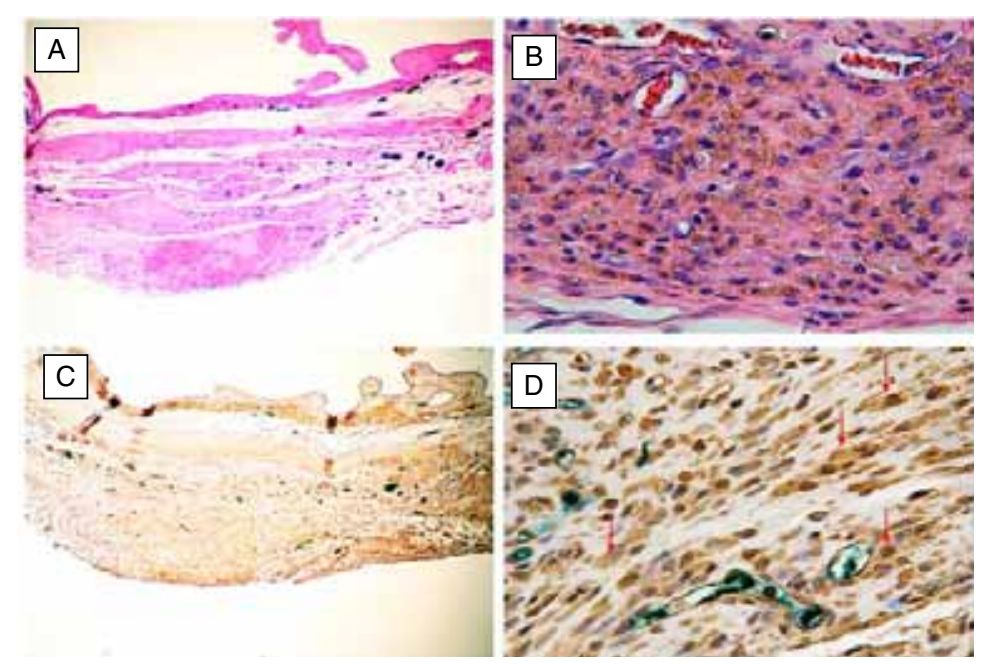

(A) $(\times 40)$ and (B) $(\times 400)$ are H\&E staining of the boxed area in Figure 1G. (C) $(\times 40)$ and (D) $(\times 400)$ are the immunohistochemical staining for sacromeric actin of the boxed area in Figure 1G. Note that there are numerous cells within the scar area, and that these cells are sacromeric actin-positive staining (red arrows).

MSC s expressed myogenic markers at 1 week after injection [13]. Shake and colleagues injected autologous M SC sinto myocardial infarction in a pig model [14]. The transplanted M SC s expressed muscle-specific proteins, assessed by immunohistochemistry at 4 weeks after injection. M oreover, the MSC therapy attenuated contractile dysfunction and pathological thinning after myocardial infarction in this model. These results suggested that M SC s may be used for cellular cardiomyoplasty to augment the function of diseased myocardium. $\mathrm{H}$ owever, the mechanism by which bone marrow-derived M SC therapies improve cardiac function remains controversial. Recent studies have highlighted the potential paracrine effects of grafted M SCS in the infarcted myocardium. Grafted bone marrow-derived cells may release paracrine factors to improve cardiac function. Kamihata and colleagues injected bone marrow-derived mononuclear cells into the infarcted myocardium in a swine myocardial infarction model [15]. Grafted cells significantly upregulated the expression of basic fibroblast growth factor, vascular endothelial growth factor and angiopoietin in the myocardium, and significantly improved regional blood flow, capillary density and cardiac function. Tang and colleagues injected autologous M SC s directly into the peri-infarct zone in a rat myocardial model [16]. At 2 weeks after injection, the expression of basic fibroblast growth factor, vascular endothelial growth factor and stem cell homing factor significantly increased, and the pro-apoptotic protein Bax decreased, assessed by western blot analysis in the ischemic myocardium. These findings were accompanied by an increase in capillary density and improvement in cardiac function. These results suggested that a paracrine action of the engrafted cells might be the major mechanism by which cardiac function is improved by M SC transplantation therapy. In order to provide evidence for the hypothesis that the secreted soluble paracrine cytoprotective factors of MSCs play a crucial role in the cardiac function improvement, $G$ necchi and colleagues collected the conditioned medium from cultured bone marrow-derived M SC s overexpressing Akt (Akt-M SCs), and demonstrated that the AktMSC-conditioned medium markedly inhibits hypoxia-induced apoptosis and triggers vigorous spontaneous contraction of adult rat cardiomyocytes in vitro [17]. The Akt-M SC-conditioned medium significantly limited infarct size and improved ventricular function after intramyocardial injection into infarcted hearts. These data support the hypothesis of paracrine mechanisms of myocardial protection and functional improvement in M SC transplantation therapy.

In our present study, we also observed that conditioned medium and M SC treatment significantly improved cardiac function compared with the saline-treated group, which was consistent with the reported results in Gnecchi's study [17]. The interesting and somewhat surprising finding of this study is that treatment with serum-containing fresh medium also improved cardiac function. O ne of the explanations is that the fetal bovine serum is a complex mixture of a large number of unknown constituents, including a broad spectrum of macromolecules, carrier proteins for lipoid substances and trace elements, attachment and spreading factors, low molecular weight nutrients, hormones and growth factors $[18,19]$. The hormones and growth factors contained in the fresh medium might work as paracrine factors to improve cardiac function. Another possible explanation is that the injected fresh medium provides essential nutrients required by injured cells within the ischemic area. This was supported by the fact that the M SC s that were suspended in the fresh medium significantly increased the scar thickness compared with the saline group. By contrast, our previous study demonstrated that MSCs that were suspended in saline did not increase scar thickness in the same myocardial infarction model [5]. 
It is likely that the increase in cell number and wall thickness in the cell transplant group was due to an increased mass of the wall of the scar due to the cells themselves, coupled with an

\section{Executive summary}

- Bone marrow-derived mesenchymal stem cells (M SCs) are multipotent cells and a potentially useful cell source for cell transplantation therapy of damaged myocardium.

- Paracrine action of the engrafted MSCs might be the major mechanism by which cardiac function is improved by MSCs transplantation therapy.

- This study demonstrates that serum-containing culture medium has a similar beneficial effect on cardiac function as conditioned medium or MSCs in rat myocardial infarction.

- The hormones and growth factors contained in the fresh medium might work as paracrine factors to improve cardiac function. improved survival rate by implanting them with fresh medium (rather than in saline as performed in our previous study [5]).

In summary, this study demonstrates that serum-containing culture medium has a similar beneficial effect on cardiac function as conditioned medium or MSCs in rat myocardial infarction. The mechanism of the benefit is unclear. Since many commercially available serums are of a high uniform quality and contain ill-defined components, future studies are needed to define the factors within the culture medium that can improve the cardiac function of the infarcted heart.

Acknowledgements

This study was supported by the $\mathrm{N}$ ational Institute of $\mathrm{H}$ ealth 1R01-H L073709.

\section{Bibliography}

1. Pittenger M F, M artin BJ : M esenchymal stem cells and their potential as cardiac therapeutics. Circ. Res 95(1), 9-20 (2004).

2. Dai $W, H$ ale $S L, K$ Koner RA: Stem cell transplantation for the treatment of myocardial infarction. Transpl. Immunol. 15(2), 91-97 (2005).

3. Piao H, Youn TJ, Kwon JS et al.: Effects of bone marrow derived mesenchymal stem cells transplantation in acutely infarcting myocardium. Eur. J. H eart Fail. 7(5), 730-738 (2005).

4. Tang $Y L$, Z hao Q, Zhang YC et al.: Autologous mesenchymal stem cell transplantation induce VEGF and neovascularization in ischemic myocardium. Regul. Pept. 117(1), 3-10 (2004).

5. Dai W, H ale SL, M artin BJ et al.: Allogeneic mesenchymal stem cell transplantation in postinfarcted rat myocardium: short- and long-term effects. Circulation 112(2), 214-223 (2005).

6. NIH publication. Guide for the Careand U se of Laboratory Animals. N ational Academy press, Washington DC,USA (1996).

7. H aynesworth SE, Goshima J, G oldberg VM, Caplan Al: Characterization of cells with osteogenic potential from human marrow. Bone 13, 81-88 (1992).
8. Pittenger M F, M ackay AM, Beck SC et al.: M ultilineage potential of adult human mesenchymal stem cells. Science 284 143-147 (1999).

9. Hochman JS, Choo H : Limitation of myocardial infarct expansion by reperfusion independent of myocardial salvage. Circulation 75(1), 299-306 (1987).

10. Fuchs S, Baffour R, Zhou YF et al.: Transendocardial delivery of autologous bone marrow enhances collateral perfusion and regional function in pigs with chronic experimental myocardial ischemia. J. Am. Coll. Cardiol. 37(6), 1726-1732 (2001).

11. Silva GV, Perin EC, D ohmann H F et al.: Catheter-based transendocardial delivery of autologous bone-marrow-derived mononuclear cells in patients listed for heart transplantation. Tex. H eart Inst. J. 31(3), 214-219 (2004).

12. M akino S, Fukuda $K, M$ iyoshi $S$ et al.: Cardiomyocytes can be generated from marrow stromal cells in vitro. J. Clin. Invest. 103(5), 697-705 (1999).

13. Toma C, Pittenger M F, Cahill KS, Byrne BJ, Kessler PD : H uman mesenchymal stem cells differentiate to a cardiomyocyte phenotype in the adult murine heart. Circulation 105(1), 93-98 (2002).
14. Shake JG, Gruber PJ, Baumgartner WA et al.: M esenchymal stem cell implantation in a swine myocardial infarct model: engraftment and functional effects. Ann. Thorac. Surg. 73(6), 1919-1925 (2002).

15. Kamihata $\mathrm{H}, \mathrm{M}$ atsubara $\mathrm{H}, \mathrm{N}$ ishiue $\mathrm{T}$ et al.: Implantation of bone marrow mononuclear cells into ischemic myocardium enhances collateral perfusion and regional function via side supply of angioblasts, angiogenic ligands, and cytokines. Circulation 104(9), 1046-1052 (2001).

16. Tang $Y L, Z$ hao $Q, Q$ in $X$ et al.: Paracrine action enhances the effects of autologous mesenchymal stem cell transplantation on vascular regeneration in rat model of myocardial infarction. Ann. Thorac. Surg. 80(1), 229-236 (2005).

17. Gnecchi $\mathrm{M}, \mathrm{HeH}, \mathrm{N}$ oiseux $\mathrm{N}$ et al.: Evidence supporting paracrine hypothesis for Akt-modified mesenchymal stem cellmediated cardiac protection and functional improvement. FASEB J. 20(6), 661-669 (2006).

18. G straunthaler $\mathrm{G}$ : Alternatives to the use of fetal bovine serum: serum-free cell culture. ALTEX 20(4), 275-281 (2003).

19. $H$ eng $B C, H$ aider $H K h$, Sim EK, Cao T, $\mathrm{N} \mathrm{g} \mathrm{SC}$ : Strategies for directing the differentiation of stem cells into the cardiomyogenic lineage in vitro. Cardiovasc. Res. 62(1), 34-42 (2004). 